ARTICLE

Molecular Diagnostics

\title{
Prognostic role of carcinoembryonic antigen and carbohydrate antigen 19-9 in metastatic colorectal cancer: a $B R A F$-mutant subset with high CA 19-9 level and poor outcome
}

\author{
Maria Thomsen ${ }^{1,2}$, Eva Skovlund ${ }^{3}$, Halfdan Sorbye ${ }^{4}$, Nils Bolstad ${ }^{5}$, Kjell Johannes Nustad ${ }^{5}$, Bengt Glimelius ${ }^{6}$, Per Pfeiffer ${ }^{7,8}$, Elin H. Kure ${ }^{9}$,
} Julia S. Johansen ${ }^{10}$, Kjell Magne Tveit ${ }^{1,2,11}$, Thoralf Christoffersen ${ }^{12}$ and Tormod Kyrre Guren ${ }^{1,11}$

BACKGROUND: Mutation status of RAS and BRAF, as well as serum levels of carcinoembryonic antigen (CEA) and carbohydrate antigen 19-9 (CA 19-9), are biomarkers used in clinical management of patients with gastrointestinal cancers. This study aimed to examine the prognostic role of these biomarkers in a patient population that started first-line chemotherapy for unresectable metastatic colorectal cancer (mCRC) in the NORDIC-VII study.

METHODS: CEA and CA 19-9 were measured in serum samples from 545 patients obtained before the start of chemotherapy. Four hundred and ninety-four patients had detectable levels of carbohydrate antigen 19-9 (CA 19-9). RAS (exons 2-4) and BRAF (V600E) mutation status were available from 440 patients. Overall survival (OS) was estimated in patient groups defined by serum CEA or CA 19-9 levels using cut-off values of $5 \mu \mathrm{g} / \mathrm{L}$ and $35 \mathrm{kU} / \mathrm{L}$, respectively, in the total population and in subgroups according to RAS and BRAF mutation status.

RESULTS: For both CEA and CA 19-9, elevated serum levels were associated with reduced OS in adjusted analyses which included RAS and BRAF mutation status, baseline World Health Organization performance status, and levels of alkaline phosphatase and Creactive protein. The negative prognostic information provided by an elevated CA 19-9 level was particularly marked in patients with $B R A F$ mutation (hazard ratio $=4.35$, interaction $P=0.003$, in an adjusted model for OS).

CONCLUSIONS: High baseline serum concentrations of CEA and CA 19-9 provide independent information of impaired prognosis in $\mathrm{mCRC}$. In patients with BRAF-mutant tumours, elevated serum CA 19-9 may identify a subgroup with highly aggressive disease and could contribute to improving therapeutic decisions.

British Journal of Cancer https://doi.org/10.1038/s41416-018-0115-9

\section{INTRODUCTION}

Colorectal cancer (CRC) is the third most common cancer and $40-50 \%$ of patients with CRC will develop distant metastases. ${ }^{1}$ Systemic therapy is the main treatment option for metastatic CRC (mCRC), and better treatment during the past decades has resulted in improved survival. However, $\mathrm{mCRC}$ is a heterogeneous disease, and selecting patients for optimal treatment is a challenge. Many factors determine the outcome. Development of robust and easily available biomarkers may help to individualise treatment.

Mutations in RAS (exons 2-4) and particularly BRAF (V600E) in the tumour cells are associated with impaired prognosis, ${ }^{2-6}$ and mutation status is routinely used in the clinical management of patients with mCRC. About $50 \%$ of the patients have RAS mutations which predict lack of effect from systemic treatment with epidermal growth factor receptor (EGFR) antibodies. ${ }^{7,8}$ The activating BRAF (V600E) mutation is found in $5-20 \%$ of the tumours in $\mathrm{mCRC}$ patient cohorts. ${ }^{3,5,6,9}$

Assays of certain glycoproteins and carbohydrates expressed by cancer cells are used in the clinical management of patients with gastrointestinal malignancies. ${ }^{10,11}$ Carcinoembryonic antigen (CEA), a glycoprotein belonging to a group of adhesion molecules, is produced in the epithelium of the large intestine and may be involved in malignancy. ${ }^{12}$ CEA has an established role as a biomarker in diagnosis, treatment and surveillance in $\mathrm{CRC}^{10}$ and elevated serum levels of CEA are associated with inferior prognosis. ${ }^{10,13,14}$ Carbohydrate antigen 19-9 (CA 19-9), a tetrasaccharide carbohydrate also termed sialyl Lewis a, synthesised by gastrointestinal epithelium, is an established serum biomarker for monitoring treatment of patients with pancreatic cancer. While

\footnotetext{
${ }^{1}$ Department of Oncology, Oslo University Hospital, Oslo, Norway; ${ }^{2}$ Institute of Clinical Medicine, Faculty of Medicine, University of Oslo, Oslo, Norway; ${ }^{3}$ Department of Public Health and Nursing, Norwegian University of Science and Technology, Trondheim, Norway; ${ }^{4}$ Department of Oncology, Haukeland University Hospital and Department of Clinical Science, University of Bergen, Bergen, Norway; ${ }^{5}$ Department of Medical Biochemistry, Oslo University Hospital, Oslo, Norway; ${ }^{6}$ Department of Immunology, Genetics and Pathology, Uppsala University, Uppsala, Sweden; ${ }^{7}$ Department of Oncology, Odense University Hospital, Odense, Denmark; ${ }^{8}$ Institute of Clinical Research, University of Southern Denmark, Odense, Denmark; ${ }^{9}$ Department of Cancer Genetics, Institute for Cancer Research, Oslo University Hospital, Oslo, Norway; ${ }^{10}$ Departments of Oncology and Medicine, Herlev and Gentofte Hospital, Copenhagen University Hospital, Copenhagen, Denmark; ${ }^{11}$ K.G.Jebsen Colorectal Cancer Research Centre, Oslo University Hospital, Oslo, Norway and ${ }^{12}$ Department of Pharmacology, Institute of Clinical Medicine, Faculty of Medicine, University of Oslo, Oslo, Norway

Correspondence: Tormod Kyrre Guren (uxtour@ous-hf.no)
}

Received: 29 September 2017 Revised: 26 March 2018 Accepted: 16 April 2018

Published online: 06 June 2018 


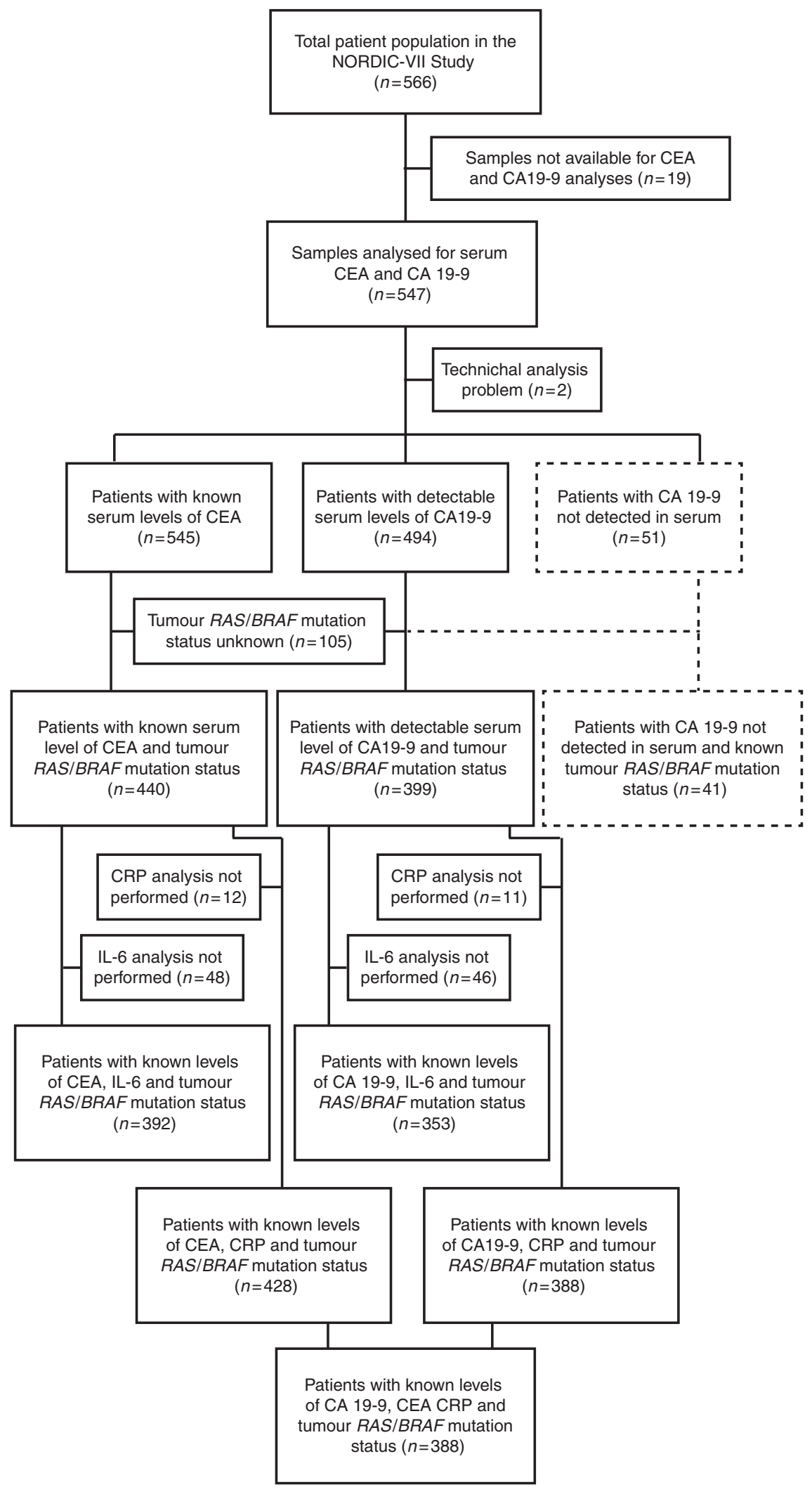

Fig. 1 CONSORT diagram. Study populations

there is some evidence of a relationship between elevated CA 199 levels and outcome in $\mathrm{CRC}^{15-17}$ its role in the management of patients with mCRC is so far unclear. ${ }^{10,18}$ Little is also known about the prognostic information that can be obtained from the serum levels of these two biomarkers when analysed in relation to tumour RAS and BRAF mutation status.

One particular focus of the present study is the group of patients with BRAF-mutant tumours. Although the presence of 


\begin{tabular}{|c|c|c|c|c|c|c|}
\hline & $\begin{array}{l}\text { Total }(n= \\
566)\end{array}$ & $\begin{array}{l}\text { CEA/CA } 19-9 \\
\text { analysed }(n=545)\end{array}$ & $\begin{array}{l}\text { CA 19-9 detected } \\
(n=494)\end{array}$ & $\begin{array}{l}\text { CA 19-9 not } \\
\text { detected }(n=51)\end{array}$ & $\begin{array}{l}\text { CEA analysed } R A S / B R A F \\
\text { analysed }(n=440)\end{array}$ & $\begin{array}{l}\text { CA } 19-9 \text { detected RAS/ } \\
\text { BRAF analysed }(n=399)\end{array}$ \\
\hline \multicolumn{7}{|l|}{ Age (years) } \\
\hline Median (min, max) & $62(24,75)$ & $62(24,75)$ & $62(24,75)$ & $60(30,75)$ & $62(24,75)$ & $62(24,75)$ \\
\hline Female & $232(41)$ & $223(41)$ & $202(41)$ & $21(41)$ & $175(40)$ & $158(40)$ \\
\hline \multicolumn{7}{|c|}{ WHO performance status, $n(\%)$} \\
\hline 0 & $380(67)$ & $367(67)$ & $328(66)$ & $39(76)$ & $295(67)$ & $263(67)$ \\
\hline 1 & $162(29)$ & $155(28)$ & $145(29)$ & $10(20)$ & $126(29)$ & $119(29)$ \\
\hline 2 & $24(4)$ & $23(4)$ & $21(4)$ & $2(4)$ & $19(4)$ & $17(4)$ \\
\hline \multicolumn{7}{|l|}{ Previous surgery, $n$ (\%) } \\
\hline $\begin{array}{l}\text { Primary tumour } \\
\text { resected }\end{array}$ & $382(67)$ & $369(68)$ & $334(68)$ & $35(69)$ & $335(76)$ & $303(76)$ \\
\hline $\begin{array}{l}\text { Intact primary } \\
\text { tumour }\end{array}$ & $184(33)$ & $176(32)$ & $160(32)$ & $16(31)$ & $105(24)$ & $96(24)$ \\
\hline \multicolumn{7}{|l|}{ Prior pelvic RT, $n$ (\%) } \\
\hline Yes & $80(14)$ & $75(14)$ & $68(14)$ & $7(14)$ & $66(15)$ & $60(15)$ \\
\hline No & $486(86)$ & $470(86)$ & $426(86)$ & $44(86)$ & $374(85)$ & $339(85)$ \\
\hline \multicolumn{7}{|l|}{ Prior adjuvant $\mathrm{CT}, n(\%)$} \\
\hline Yes & $51(9)$ & $49(9)$ & $42(9)$ & $7(14)$ & $44(10)$ & $38(10)$ \\
\hline No & $515(91)$ & $496(91)$ & $452(91)$ & $44(86)$ & $396(90)$ & $351(90)$ \\
\hline Normal & $298(53)$ & $286(52)$ & $258(48)$ & $26(55)$ & $240(55)$ & $217(54)$ \\
\hline$>\mathrm{UNL}$ & $268(47)$ & $259(48)$ & $236(48)$ & $23(45)$ & $200(45)$ & $182(46)$ \\
\hline \multicolumn{7}{|l|}{ Platelet count, $n$ (\%) } \\
\hline$\leq 400 / \mathrm{nL}$ & $398(70)$ & $383(70)$ & $347(70)$ & $36(71)$ & $315(72)$ & $287(72)$ \\
\hline$>400 / \mathrm{nL}$ & $168(30)$ & $162(30)$ & $147(30)$ & $15(29)$ & $125(28)$ & $112(28)$ \\
\hline \multicolumn{7}{|c|}{ White blood cell count, $n$ (\%) } \\
\hline$\leq 10 / \mathrm{nL}$ & $428(76)$ & $416(76)$ & $369(75)$ & $44(86)$ & $345(78)$ & $309(77)$ \\
\hline$>10 / \mathrm{nL}$ & $138(24)$ & $132(24)$ & $125(25)$ & $7(14)$ & $95(22)$ & $90(23)$ \\
\hline \multicolumn{7}{|c|}{ RAS/BRAF mutation status, $n$ (\%) } \\
\hline$R A S / B R A F$ wild-type & & & & $15^{\mathrm{a}}(37)$ & $186(42)$ & $171(43)$ \\
\hline RAS mutation & & & & $22^{\mathrm{a}}(54)$ & $201(46)$ & $179(45)$ \\
\hline BRAF mutation & & & & $4^{\mathrm{a}}(10)$ & $53(12)$ & $49(12)$ \\
\hline \multicolumn{7}{|l|}{ CRP level, $n$ (\%) } \\
\hline$\leq 10 \mathrm{mg} / \mathrm{L}$ & & & & $18^{\mathrm{b}}(45)$ & $194^{d}(45)$ & $176^{f}(45)$ \\
\hline$>10 \mathrm{mg} / \mathrm{L}$ & & & & $22^{\mathrm{b}}(55)$ & $234^{\mathrm{d}}(55)$ & $212^{f}(55)$ \\
\hline \multicolumn{7}{|l|}{ IL-6 level, $n$ (\%) } \\
\hline$<5.6 \mathrm{ng} / \mathrm{L}$ & & & & $21^{c}(54)$ & $193^{\mathrm{e}}(49)$ & $172^{9}(49)$ \\
\hline$\geq 5.6 \mathrm{ng} / \mathrm{L}$ & & & & $18^{\mathrm{c}}(46)$ & $199^{\mathrm{e}}(51)$ & $181^{\mathrm{g}}(51)$ \\
\hline
\end{tabular}


BRAF mutation in general is known to be associated with an inferior outcome of $\mathrm{mCRC},{ }^{2,4,6}$ there is a significant heterogeneity within the BRAF-mutant population., ${ }^{9,20}$ It is important to learn more about the basis for this heterogeneity, since it is conceivable that optimal therapeutic care might differ for these patients. ${ }^{20,21}$ We have previously shown that in $\mathrm{mCRC}$ patients with BRAF mutations, evidence of a systemic inflammation predicted an extremely aggressive disease with very short survival. ${ }^{22}$ Furthermore, during treatment of $\mathrm{mCRC}$ patients, we have accidentally observed high serum levels of CA 19-9 in several patients with $B R A F$-mutant tumours. The aim of the present study was to explore the prognostic information of serum CEA and CA 19-9 in patients with mCRC, especially as related to RAS and BRAF mutation status, and with emphasis on CA 19-9 in patients with BRAF-mutant tumours.

\section{MATERIALS AND METHODS}

Patients

The randomised NORDIC-VII study investigated the effects of combining cetuximab with the Nordic FLOX regimen with bolus 5flououracil/folinic acid and oxaliplatin ${ }^{23}$ in previously untreated patients with unresectable mCRC. ${ }^{24} \mathrm{~A}$ total of 566 patients were randomly assigned to receive FLOX, cetuximab plus FLOX or cetuximab combined with intermittent FLOX as first-line palliative chemotherapy. There were no statistically significant differences in outcome (progression-free survival (PFS), overall survival (OS), overall response rate or secondary R0 resection of metastases) between the different treatment arms in the NORDIC-VII study., 24 In the present study, data were analysed across the three treatment arms.

The CONSORT diagram in Fig. 1 describes the different patient populations. CA $19-9$ and CEA were analysed in serum from 545 patients. Tumour RAS/BRAF mutation status was known in 440 of these patients, and further information about plasma C-reactive protein (CRP) and interleukin-6 (IL-6) level was available in 428 and 392 patients, respectively. The different prognostic clinicopathological markers were defined as alkaline phosphatase (ALP) level normal or elevated (above upper normal limit (UNL) based on institutional reference values at the study sites), CRP (below or above $10 \mathrm{mg} / \mathrm{L}$ ), platelet count (below or above $400 / \mathrm{nL}$ ) and white blood cell count (below or above 10/nL). These cut-off values have been used in large mCRC phase III trials and in prognostic indexes. $3,8,25,26$ The baseline demographics and clinical characteristics of the patients are shown in Table 1.
Mutation analyses of KRAS, NRAS and BRAF

Genomic DNA was extracted from formalin-fixed paraffinembedded $10 \mu \mathrm{m}$ tumour tissue sections (65-70\% (median) tumour cells) using QIAamp DNA Micro Kit (Cat. 56304, Qiagen). Tumour DNA was screened for the presence of RAS (exons 2-4) and BRAF (V600E) mutations as previously described. 6,24

\section{Measurement of CEA and CA 19-9}

Fresh-frozen serum samples were obtained from patients before the start of treatment and after 8 weeks of treatment. The samples were thawed and mixed before analysis of CEA (Ref. No. 04491777 190) and CA 19-9 (Ref. No. 11776193 122) on the Cobas e601 instrument (Roche Diagnostics $\mathrm{GmbH}$, Mannheim, Germany). Established Norwegian reference values were used, with cut-off values $5 \mu \mathrm{g} / \mathrm{L}$ for CEA and $35 \mathrm{kU} / \mathrm{L}$ for CA 19-9. Patients with undetectable levels of CA 19-9 (i.e., below the lower analytical detection limit; $5 \mathrm{kU} / \mathrm{L}$ ) were classified as a separate category in the primary statistical analyses. The results are presented in accordance with the REMARK guidelines. ${ }^{27}$

\section{Statistical analyses}

The statistical analyses were performed using IBM SPSS (version 23, IBM Corp., Armonk, NY, USA). Demographic data were described with median and range (continuous variables) or with proportions and percentages (categorical variables). The prognostic values of different serum levels of CEA and CA 19-9 and survival were assessed by Kaplan-Meier method, log-rank test and Cox proportional hazards model. Separate analyses of the effect of World Health Organization (WHO) performance status, ALP level, number of metastatic sites, CRP level and tumour RAS/BRAF mutation status were performed. Only variables statistically significant $(P<0.05)$ in these analyses were included in the multivariable analyses, and models were restricted to include statistically significant variables only. An interaction term was included in the Cox model to explore the effect of mutation status on the prognostic effect of serum levels of CEA and CA 19-9.

\section{RESULTS}

Clinical characteristics and serum levels of CEA and CA 19-9

The frequency distribution of the levels of CEA and CA 19-9 is shown in Fig. 2a, b, respectively. From 545 analysed patients, 96 (18\%) had serum levels of CEA below $5 \mu \mathrm{g} / \mathrm{L}$ and 449 (82\%) had elevated CEA levels ( $\geq 5 \mu \mathrm{g} / \mathrm{L}$ ). Furthermore, 494 patients had detectable serum levels of CA 19-9 in their serum samples. Of these patients, 188 (38\%) had a serum level of CA 19-9 below 35
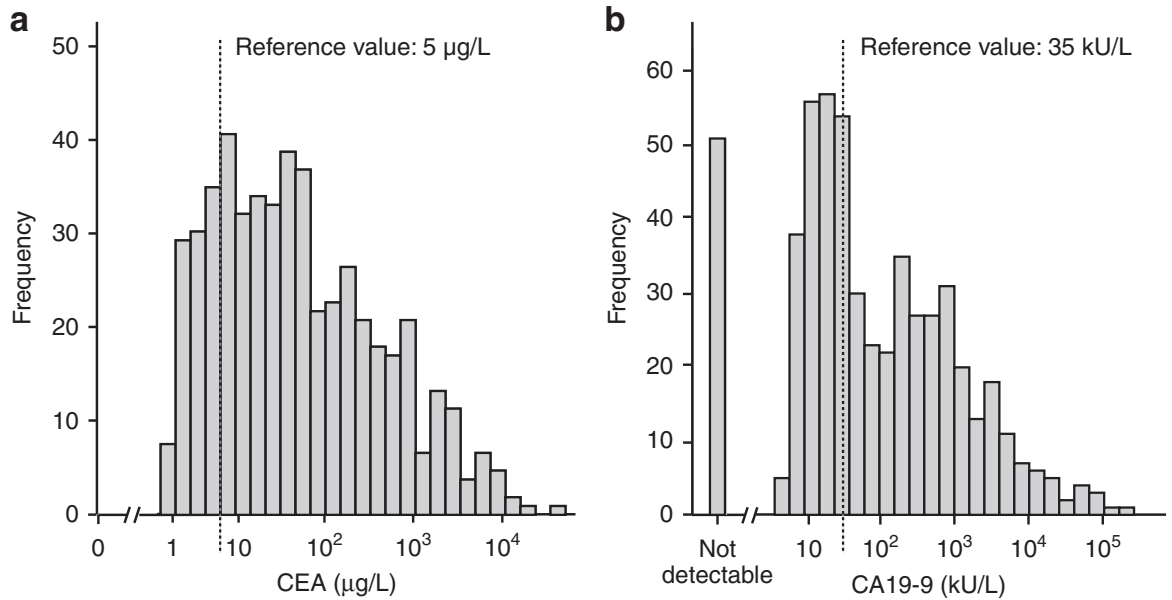

Fig. 2 Histograms. Distribution of serum level of (a) CEA (median: $36 \mu \mathrm{g} / \mathrm{L}$; range: $0.5-32,916 \mu \mathrm{g} / \mathrm{L}$ ) and (b) CA 19-9 (median $50 \mathrm{kU} / \mathrm{L}$; range $5-185,991 \mathrm{kU} / \mathrm{L}$ ). Fifty-one patients had undetectable levels of CA $19-9$ in the serum 

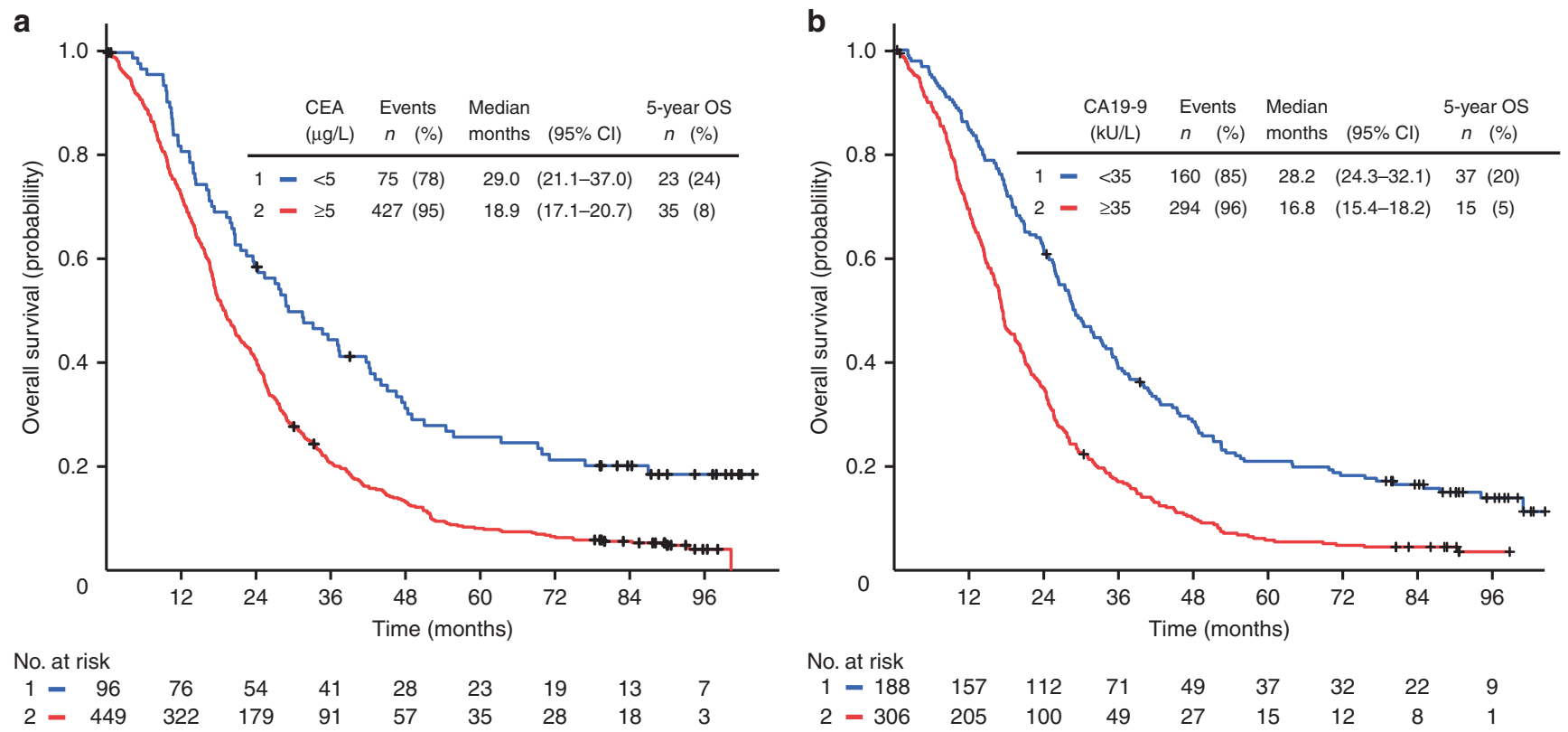

d
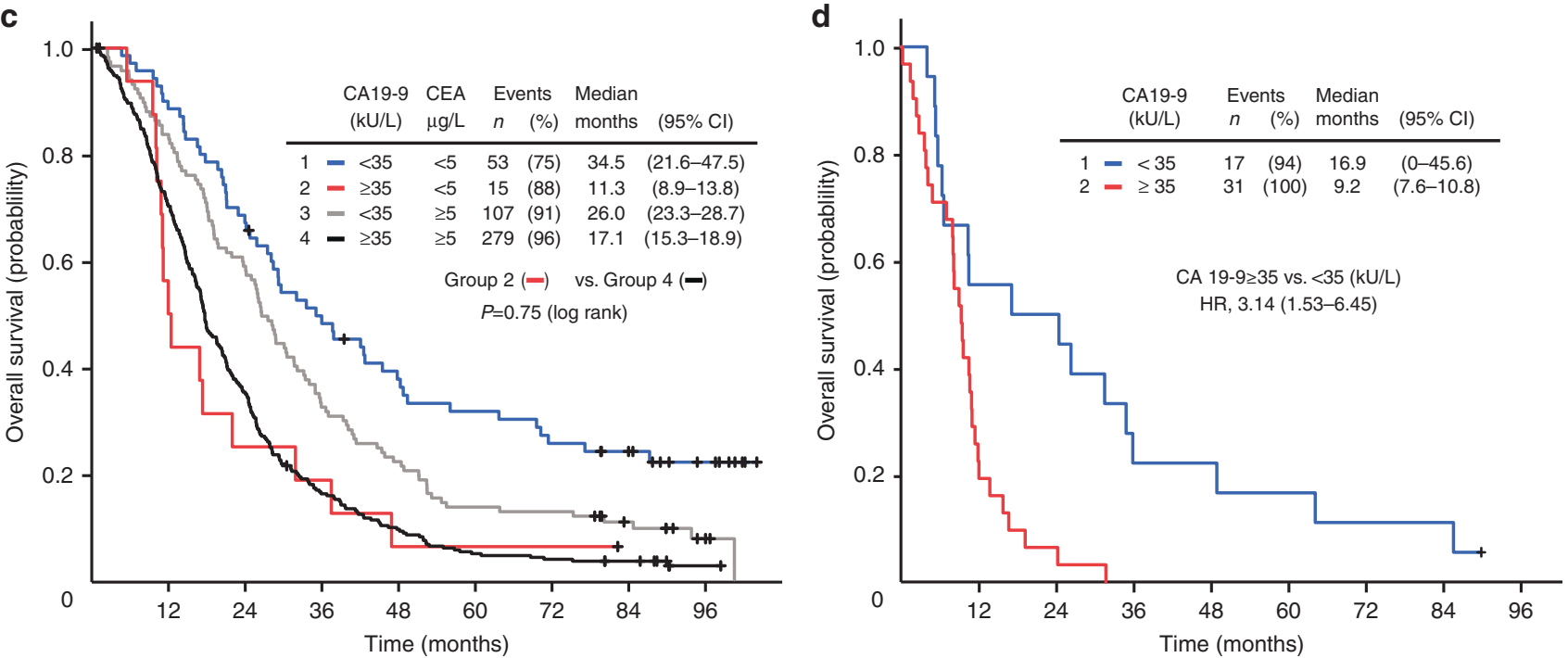

$\begin{array}{lccccccccc}\text { No. at risk } & & & & & & & \\ 1- & 71 & 62 & 45 & 33 & 24 & 21 & 17 & 13 & 7 \\ 2- & 17 & 7 & 4 & 3 & 1 & 1 & 1 & - & - \\ 3- & 117 & 95 & 67 & 33 & 25 & 16 & 15 & 9 & 2 \\ 4- & 289 & 198 & 96 & 46 & 26 & 14 & 11 & 8 & 1\end{array}$

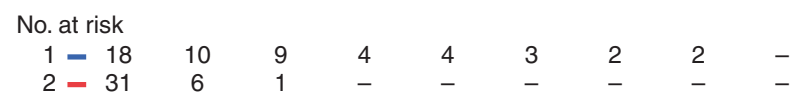

Fig. 3 Overall survival. OS in 545 and 494 patients with different baseline serum levels of CEA and CA 19-9, respectively (a-c). a Serum level of CEA $<5 \mu \mathrm{g} / \mathrm{L}$ or CEA $\geq 5 \mu \mathrm{g} / \mathrm{L}$. b Serum level of CA $19-9<35 \mathrm{kU} / \mathrm{L}$ or CA $19-9 \geq 35 \mathrm{kU} / \mathrm{L}$. c Serum level of CA 19-9 level <35 kU/L or CA $19-9 \geq 35$ $\mathrm{kU} / \mathrm{L}$ in patients with CEA level $<5 \mu \mathrm{g} / \mathrm{L}$ or CEA $\geq 5 \mu \mathrm{g} / \mathrm{L}$. d OS in 51 patients with BRAF-mutant tumours; CA 19-9 level $<35 \mathrm{kU} / \mathrm{L}$ or CA $19-9 \geq 35$ $\mathrm{kU} / \mathrm{L}$. OS overall survival, $H R$ hazard ratio, $C$ confidence interval

$\mathrm{kU} / \mathrm{L}$ and 306 (62\%) had elevated CA 19-9 ( $\geq 35 \mathrm{kU} / \mathrm{L})$. From 51 patients (9\%), CA 19-9 was below the detection limit.

For both CEA and CA 19-9, elevated serum levels were associated with impaired baseline clinical patient characteristics, such as inferior WHO performance status, intact primary tumour, synchronous metastases, elevated ALP level, elevated platelet $(>400 / \mathrm{nL})$ and white blood cell $(>10 / \mathrm{nL})$ counts and elevated CRP (>10 mg/L) (Supplementary Table 1). Elevated CA 19-9 was somewhat more frequent in patients with colon cancer as compared to rectal cancer, while there was no association between CEA level and origin of primary tumour. Furthermore, $33 \%$ and $71 \%$ of the patients with lung-only metastases had levels of CEA below $5 \mu \mathrm{g} / \mathrm{L}$ and CA $19-9$ below $35 \mathrm{kU} / \mathrm{L}$, respectively. No relationship between other metastatic sites and serum levels of CEA or CA 19-9 was observed.

In patients with elevated serum CA $19-9$ ( $\geq 35 \mathrm{kU} / \mathrm{L})$, the median was numerically higher in those with with $B R A F$-mutant tumours $(853 \mathrm{kU} / \mathrm{L})$ compared to those with RAS-mutant or RAS/BRAF wildtype tumours ( 336 and $254 \mathrm{kU} / \mathrm{L}$, respectively). Furthermore, 8 of the 16 patients with an isolated elevated CA 19-9 (i.e., CEA not increased) had BRAF-mutant tumours.There was no difference in median CEA value between subgroups based on RAS or BRAF mutation status.

Serum level of CEA as an independent prognostic biomarker As expected, there was a relationship between increasing serum levels of CEA (grouped in quartiles) and impaired survival (log-rank 
test for trend, $P<0.001)$. Patients with elevated CEA $(\geq 5 \mu \mathrm{g} / \mathrm{L})$ and low CEA $(<5 \mu \mathrm{g} / \mathrm{L})$ had a median OS of 19 and 29 months, respectively (hazard ratio $(\mathrm{HR}), 1.85$ (95\% confidence interval $(\mathrm{Cl})$ 1.45-2.38); $P<0.001)$. Furthermore, $24 \%$ of the patients with serum CEA below $5 \mu \mathrm{g} / \mathrm{L}$ survived at least 5 years compared to $8 \%$ of those with an elevated CEA level (Fig. 3a), although there was no difference in frequency of secondary metastasectomy in the two subgroups ( $9 \%$ and $8 \%$, respectively). The prognostic information of baseline CEA was further confirmed in an adjusted model including other prognostic markers and clinical characteristics believed to be potential confounders, including $\mathrm{WHO}$ performance status, ALP and CRP levels and RAS/BRAF mutation status (adjusted HR, $1.78(95 \% \mathrm{Cl} 1.34-2.37) ; P<0.001)$, as shown in Supplementary Table 2.

\section{Serum CA 19-9 level as a prognostic biomarker}

There was a statistically significant relationship between levels of CA 19-9 (grouped in quartiles) and OS (log-rank test for trend, $P<0.001)$. Figure $3 \mathrm{~b}$ shows that an elevated serum CA 19-9 $(\geq 35$ $\mathrm{kU} / \mathrm{L}$ ) at baseline was associated with impaired outcome, with median OS 17 months, compared to patients with CA 19-9 below $35 \mathrm{kU} / \mathrm{L}$ who had a median OS of 28 months (HR $=1.95(95 \% \mathrm{Cl}$ $1.60-2.38) ; P<0.001)$. Thirty-seven (20\%) of the patients with serum CA $19-9$ level below $35 \mathrm{kU} / \mathrm{L}$ survived beyond 5 years, compared to $5 \%$ of the patients with elevated CA 19-9, with no difference in resection rates of metastases in the two groups (10\% and $8 \%$, respectively). This association between elevated CA 19-9 and impaired OS was found both in patients with low and elevated serum level of CEA (Fig. 3c). Essentially, a similar association was demonstrated in the groups of patients with known RAS/BRAF mutation status or RAS/BRAF mutation and plasma CRP level (not shown). There was a trend that the prognostic value of elevated CA 19-9 was more pronounced in patients with colon cancer compared to rectal cancer, but no statistically significant interaction between CA 19-9 and site on OS was detected (not shown).

The baseline serum level of CA 19-9 or CEA did not influence the initial chemotherapy response, measured as rates of overall response, disease control, direct progression or secondary resection of metastases, neither in the total patient population nor in subgroups defined by RAS or BRAF mutations (Supplementary Tables 3 and 4).

Paired serum samples obtained before the start of treatment and after four cycles of chemotherapy (approximately 8 weeks of treatment) were available from 384 and 260 patients with elevated baseline levels of CEA and CA 19-9, respectively. There was an association between treatment response and a drop in serum levels for both markers (Supplementary Table 5). However, the change, absolute or relative, in serum levels of CEA and CA 199 did not predict prognosis better than the baseline level before the start of treatment (not shown).

For 51 patients (9\%), CA 19-9 was not detectable in the serum samples as judged by the lower reference value. However, in this subgroup 33 of the patients (6\% of the analysed population) had no analytic signal or very low values classified as analytic noise, whereas 18 patients had CA $19-9$ values between 3 and $5 \mathrm{kU} / \mathrm{L}$, below the lower analytical reference value. These data suggest that at least $2 / 3$ of the patients with no detectable CA 19-9 had Le (a-b-) genotype in the Lewis blood group system, consistent with the reported frequency of $5 \%$ to $10 \%$ of individuals lacking expression of Lewis antigens in Caucasian populations. ${ }^{18,28}$ The subgroup of patients for whom CA 19-9 could not be detected showed clinical features that were essentially similar to the total population (Table 1) and there was no statistically significant difference between the subcategories and clinical outcome (Supplementary Figure 1A). Sensitivity analyses including these patients with CA 19-9 assigned the value 0 did not significantly alter the results of the study (not shown).
Table 2. Association between prognostic factors and overall survival in 388 patients with detectable serum levels of CA 19-9 ${ }^{\text {a }}$

\begin{tabular}{|c|c|c|c|}
\hline Variables & $\mathrm{HR}$ & $95 \% \mathrm{Cl}$ & $P$ value \\
\hline \multicolumn{4}{|c|}{ RAS/BRAF mutation status } \\
\hline \multicolumn{2}{|c|}{$R A S / B R A F$ wild-type } & & \multirow[t]{3}{*}{$0.003^{b}$} \\
\hline CA $19-9<35 \mathrm{kU} / \mathrm{L}$ & 1 & & \\
\hline CA $19-9 \geq 35 \mathrm{kU} / \mathrm{L}$ & 1.35 & $0.96-1.91$ & \\
\hline \multicolumn{4}{|l|}{ RAS mutation } \\
\hline CA $19-9<35 \mathrm{kU} / \mathrm{L}$ & 1 & \multirow{2}{*}{\multicolumn{2}{|c|}{$1.00-2.05$}} \\
\hline CA $19-9 \geq 35 \mathrm{kU} / \mathrm{L}$ & 1.43 & & \\
\hline \multicolumn{4}{|l|}{$B R A F$ mutation } \\
\hline CA $19-9<35 \mathrm{kU} / \mathrm{L}$ & 1 & \multirow{2}{*}{\multicolumn{2}{|c|}{$2.89-8.28$}} \\
\hline CA $19-9 \geq 35 \mathrm{kU} / \mathrm{L}$ & 4.35 & & \\
\hline \multicolumn{4}{|l|}{ CEA level } \\
\hline$<5 \mu \mathrm{g} / \mathrm{L}$ & 1 & & \multirow[t]{2}{*}{0.036} \\
\hline$\geq 5 \mu \mathrm{g} / \mathrm{L}$ & 1.41 & $1.02-1.94$ & \\
\hline \multicolumn{4}{|l|}{ WHO performance status } \\
\hline 0 & 1 & & \multirow[t]{3}{*}{0.004} \\
\hline 1 & 1.37 & $1.07-1.76$ & \\
\hline 2 & 2.56 & $1.31-3.89$ & \\
\hline \multicolumn{4}{|c|}{ Alkaline phosphatase level } \\
\hline Normal & 1 & & \multirow[t]{2}{*}{0.003} \\
\hline$>\mathrm{UNL}$ & 1.44 & $1.13-1.82$ & \\
\hline \multicolumn{4}{|l|}{ CRP level } \\
\hline Per category ${ }^{c}$ & 1.17 & $1.05-1.31$ & 0.006 \\
\hline \multicolumn{4}{|c|}{$\begin{array}{l}\text { Cl confidence interval, CEA carcinoembryonic antigen, CA carbohydrate } \\
\text { antigen, } C R P \text { C-reactive protein, } H R \text { hazard ratio, OS overall survival, UNL } \\
\text { upper normal limit, WHO World Health Organization a Adjusted model } \\
\text { including the interaction between tumour } R A S / B R A F \text { mutation status and } \\
\text { CA 19-9. }{ }^{b} \text { Interaction } P{ }^{C} \text { CRP categories: from } 0 \text { to } 10 \mathrm{mg} / \mathrm{L} \text {, from } 10 \text { to } 30 \\
\mathrm{mg} / \mathrm{L} \text {, from } 30 \text { to } 60 \mathrm{mg} / \mathrm{L} \text {, from } 60 \mathrm{mg} / \mathrm{L} \text { and higher. }\end{array}$} \\
\hline
\end{tabular}

Serum levels of CA 19-9 and tumour BRAF mutation status The data showed that the prognostic information from elevated levels of CA 19-9 differed depending on the RAS and BRAF mutation status of the tumour, with a statistically significant interaction in adjusted analyses $(P=0.003)$. In contrast, there was no statistically significant interaction between serum CEA level and RAS/BRAF mutation status $(P=0.27)$.

Elevated levels of CA 19-9 were associated with a particularly impaired OS in BRAF-mutant cancers. The patients with BRAFmutant tumours had an estimated median OS of 17 and 9 months in unadjusted analyses stratified on low and elevated serum levels of CA 19-9, respectively, and the two groups differed significantly in the tail of the Kaplan-Meier curves (Fig. 3d). In an adjusted proportional hazards model including WHO performance status and levels of ALP, CEA and CRP, an elevated level of serum CA 19-9 was associated with a significant relative reduction in survival (adjusted $\mathrm{HR}=4.35$ (95\% Cl 2.89-8.28) in patients with tumours harbouring BRAF mutation (Table 2).

In the NORDIC-VII patient cohort, we previously reported that an elevated level of IL- 6 was associated with an impaired prognosis, especially in patients with BRAF-mutant tumours. ${ }^{22}$ To examine the relationship between $C A \quad 19-9$ and $I L-6$, we included information about IL-6 levels (cut-off value $5.6 \mathrm{ng} / \mathrm{L}$ ) in the analysis of how CA 19-9 predicts OS. Table 3, showing results from patients with BRAF-mutant tumours, demonstrates that at high levels of IL6 with poor survival there was still a further impaired outcome associated with high CA 19-9. This suggests that IL-6 and CA 19-9, at least partly, reflect independent mechanisms associated with inferior prognosis. 
CEA and CA 19-9 as prognostic biomarkers in mCRC

Table 3. Outcome in 42 patients with BRAF-mutant tumours: OS in subgroups defined by baseline level of CA 19-9 and IL-6

\begin{tabular}{lllll}
\hline Variables & $n$ & Events & Median OS (95\% Cl) & HR (95\% Cl) \\
\hline CA $19-9<35 \mathrm{kU} / \mathrm{L}$ IL-6 $<5.6 \mathrm{ng} / \mathrm{L}$ & 10 & 9 & $26.0(15.2-36.8)$ & $11.8(9.4-14.1)$ \\
CA $19-9 \geq 35 \mathrm{kU} / \mathrm{L}$ IL-6 $<5.6 \mathrm{ng} / \mathrm{L}$ & 7 & 7 & $6.3(5.2-7.4)$ & 1 \\
CA $19-9<35 \mathrm{kU} / \mathrm{L}$ IL-6 $\geq 5.6 \mathrm{ng} / \mathrm{L}$ & 6 & 6 & $8.1(2.3-13.9)$ & $4.3(1.3-14.0)$ \\
CA $19-9 \geq 35 \mathrm{kU} / \mathrm{L}$ IL-6 $\geq 5.6 \mathrm{ng} / \mathrm{L}$ & 19 & 19 & $4.8(1.5-14.9)$ \\
\hline IL-6 interleukin-6, CA carbohydrate antigen, Cl confidence interval, HR hazard ratio, OS overall survival & $9.0(3.0-26.9)$ \\
\hline
\end{tabular}

\section{DISCUSSION}

The present results add new information about the two serum biomarkers CEA and CA 19-9, both of which are routinely used in the management of gastrointestinal cancers. The data provide evidence that elevated serum levels of CEA and CA 19-9, measured before the start of first-line chemotherapy, are independent, negative prognostic factors in patients with mCRC.

The relationship between elevated baseline CEA and impaired prognosis is in line with previous studies on patients with metastatic disease. ${ }^{13,29,30}$ The present results indicate that CEA is an independent prognostic biomarker in $\mathrm{mCRC}$ in adjusted models including RAS/BRAF mutation status. In addition, we want to bring attention to the favourable OS and the substantial proportion of long-term survivors in the patients with a baseline serum level of CEA below $5 \mu \mathrm{g} / \mathrm{L}$.

The results showed that CA $19-9$ can also give valuable prognostic information in mCRC. Elevated CA 19-9 serum levels were associated with impaired prognosis, consistent with reported data on $\mathrm{mCRC}$ in patients with unresectable liver metastases. ${ }^{15,31}$ This was independent of the CEA level at baseline and was confirmed in adjusted analyses. It has been suggested that CA 199 could be used to monitor the disease development in $\mathrm{mCRC}$ patients who have no elevation of CEA. ${ }^{32}$ Like CEA, low levels of CA 19-9 were associated with a quite high percentage of survivors beyond 5 years.

A major finding was that the present study suggests that elevated serum CA 19-9 can have a particular role in patients with BRAF-mutant mCRC. Thus, the median CA 19-9 level was numerically high in patients with BRAF-mutant tumours and $50 \%$ of those with an isolated elevated CA 19-9 (low CEA) had BRAF-mutant tumours. More importantly, an elevated CA 19-9 level was associated with short OS within the BRAF-mutant subgroup of patients. It is known that the presence of BRAF mutation in mCRC predicts impaired outcome; ; $^{2,4,6,33}$ however, these cancers are heterogeneous. ${ }^{9,19,20}$ Although the underlying mechanisms are not clarified, the present findings suggest that $C A$ 19-9 may help identify one subgroup of BRAF-mutant mCRC patients with particularly poor prognosis. On the other hand, the measured serum CA 19-9 did not predict initial response to chemotherapy, and an elevated level was not associated with resistance to chemotherapy, neither in patients with BRAF-mutant nor non-BRAF-mutant tumours.

We recently reported an association between high levels of inflammation biomarkers and poor prognosis in $\mathrm{MCRC}$, and this was particularly prominent in the subset of patients with BRAFmutant tumours. ${ }^{22}$ Thus, in mCRC patients with BRAF-mutant tumour, unlike RAS-mutant or RAS/BRAF double wild-type tumours, high serum IL- 6 predicted markedly impaired survival. An obvious question, therefore, was whether high CA 19-9 identifies the same patients as elevated IL-6. The present data indicate that this is not the case and that IL-6 and CA 19-9 are independent biomarkers. Thus, patients with combined high CA 19-9 and high IL- 6 had extremely short survival. Together, the results strengthen the indications that BRAF-mutant $\mathrm{mCRC}$ is heterogeneous and suggest that IL-6 and CA 19-9 are biomarkers of at least partly different mechanisms that underlie aggressive disease.

One of the findings in a recent large investigation analysing the outcomes of patients with $\mathrm{MCRC}$ with BRAF mutations was that the most marked divergence between the BRAF-mutant and wildtype cancers occurred following progression on or after benefit from first-line chemotherapy. ${ }^{9}$ Our results with serum CA 19-9, CEA, IL- 6 and CRP strongly suggest that the prognostic prediction provided by these biomarkers also largely concerns advanced stages of mCRC. Thus, a marked difference in OS was noted between cases with high and low levels of the inflammatory markers despite relatively small differences in PFS. ${ }^{22}$ Furthermore, in the present study, focusing on OS, we observed that for both CEA and CA 19-9 the difference between the survival curves representing high and low levels of these markers was also reflected in long-term survival. This was so in the whole cohort and was quite dramatic when comparing low and high CA 19-9 in the subset of BRAF-mutant patients. Collectively, these data suggest that in addition to BRAF mutations, several other mechanisms, including inflammatory reactions as well as processes reflected in elevated levels of carbohydrate/glycoprotein markers, impair the outcome of $\mathrm{mCRC}$ by influencing the advanced course of the disease. These mechanisms are likely inherent in the phenotype of the particular cancer and some of them can potentiate the effect of mutated BRAF. Hopefully, better insights into these mechanisms may provide clues for developing novel therapeutic strategies for advanced mCRC.

We are aware of some limitations for CA 19-9 as a biomarker, since several benign diseases may also give rise to elevated levels, and CA 19-9 is not expressed in subjects with Le (a-b-) genotype in the Lewis blood group system. In this study, the Lewis blood-type status of the patient cohort was unknown. Nine percent of the patients in our cohort had no detectable CA 19-9 as judged by the lower reference value, and in a clinical routine setting, these patients will be reckoned as having a very low serum level of CA 19-9 below a detection limit, but it will not be possible to distinguish between patients with very low CA 19-9 levels from those who do not express CA 19-9 due to their Lewis blood type. This subgroup of patients had clinical characteristics and outcome that did not differ significantly from the total population with detectable levels of CA 19-9, suggesting that it included patients across the total population and not specifically those with a low CA 19-9 level.

Serum CA 19-9, alone or in combination with markers of systemic inflammation, can be useful in the management of patients with BRAF-mutant, primary unresectable mCRC. We believe that the findings are of general relevance, although the underlying chemotherapy regimen in the present study is not commonly used outside the Nordic countries. Elevated serum CA 19-9 level may help to identify patients with a highly aggressive disease, like those who should be considered for intensive firstline therapy, such as the triple combination FOLFOXIRI. ${ }^{4,34}$ In conclusion, integrated prognostic data, including the CEA and CA 19-9 biomarkers, may give more accurate information about the disease and can be useful in shared decision-making, enabling patient and clinician to establish an optimal treatment plan. 


\section{ACKNOWLEDGEMENTS}

We thank the patients and their families and the investigators who participated in the NORDIC-VII study. This work was supported by the Norwegian Cancer Society, the Swedish Cancer Society and by a generous financial support from the Raabe family. The NORDIC-VII study was supported by Merck KGaA, Darmstadt, Germany and Sanofi, Oslo, Norway.

\section{AUTHOR CONTRIBUTIONS}

Study design: M.T., E.S., H.S., K.M.T., T.C., T.K.G.; provision of study materials or patients: H.S., N.B., K.J.N., B.G., P.P., E.H.K., J.S.J., K.M.T., T.K.G.; data analysis and interpretation: M.T., E.S., H.S., N.B., K.J.N., K.M.T., T.C., T.K.G.; manuscript writing: all authors.

\section{ADDITIONAL INFORMATION}

Supplementary information is available for this paper at https://doi.org/10.1038/ s41416-018-0115-9.

Competing interests: The authors declare no competing interests.

Ethical approval and consent to participate: The NORDIC-VII study (http:// clinicaltrials.gov/show/NCT00145314) was approved by the National Ethics Committees and governmental authorities in each country and conducted in accordance with the Declaration of Helsinki. Prognostic biomarker substudies were part of the protocol. All patients gave written informed consent to participate in the study.

Availability of data and material: The datasets generated during and/or analysed during the current study are not publicly available but are available from the corresponding author on reasonable request.

Publisher's note: Springer Nature remains neutral with regard to jurisdictional claims in published maps and institutional affiliations.

Note: This work is published under the standard license to publish agreement. After 12 months the work will become freely available and the license terms will switch to a Creative Commons Attribution 4.0 International licence (CC BY 4.0).

\section{REFERENCES}

1. Jemal, A. et al. Global cancer statistics. CA Cancer J. Clin. 61, 69-90 (2011).

2. Tol, J., Nagtegaal, I. D. \& Punt, C. J. BRAF mutation in metastatic colorectal cancer. N. Engl. J. Med. 361, 98-99 (2009).

3. Maughan, T. S. et al. Addition of cetuximab to oxaliplatin-based first-line combination chemotherapy for treatment of advanced colorectal cancer: results of the randomised phase 3 MRC COIN trial. Lancet 377, 2103-2114 (2011).

4. Cremolini, C. et al. FOLFOXIRI plus bevacizumab versus FOLFIRI plus bevacizumab as first-line treatment of patients with metastatic colorectal cancer: updated overall survival and molecular subgroup analyses of the open-label, phase 3 TRIBE study. Lancet Oncol. 16, 1306-1315 (2015).

5. Sorbye, $H$. et al. High BRAF mutation frequency and marked survival differences in subgroups according to KRAS/BRAF mutation status and tumor tissue availability in a Prospective Population-Based Metastatic Colorectal Cancer Cohort. PLOS ONE 10, e0131046 (2015).

6. Guren, T. K. et al. Cetuximab in treatment of metastatic colorectal cancer: final survival analyses and extended RAS data from the NORDIC-VII study. Br. J. Cancer 116, 1271-1278 (2017).

7. Douillard, J. Y. et al. Panitumumab-FOLFOX4 treatment and RAS mutations in colorectal cancer. N. Engl. J. Med. 369, 1023-1034 (2013).

8. Van Cutsem, E. et al. Fluorouracil, leucovorin, and irinotecan plus cetuximab treatment and RAS mutations in colorectal cancer. J. Clin. Oncol. 33, 692-700 (2015).

9. Seligmann, J. F. et al. Investigating the poor outcomes of BRAF-mutant advanced colorectal cancer: analysis from 2530 patients in randomised clinical trials. Ann. Oncol. 28, 562-568 (2017).
10. Duffy, M. J. et al. Tumor markers in colorectal cancer, gastric cancer and gastrointestinal stromal cancers: European group on tumor markers 2014 guidelines update. Int J. Cancer 134, 2513-2522 (2014).

11. Locker, G. Y. et al. ASCO2006 update of recommendations for the use of tumor markers in gastrointestinal cancer. J. Clin. Oncol. 24, 5313-5327 (2006).

12. Beauchemin, N. \& Arabzadeh, A.. Carcinoembryonic antigen-related cell adhesion molecules (CEACAMs) in cancer progression and metastasis. Cancer Metastas. Rev. 32, 643-671 (2013).

13. Strimpakos, A. S. et al. The impact of carcinoembryonic antigen flare in patients with advanced colorectal cancer receiving first-line chemotherapy. Ann. Oncol. 21, 1013-1019 (2010).

14. Thirunavukarasu, P. et al. C-stage in colon cancer: implications of carcinoembryonic antigen biomarker in staging, prognosis, and management. J. Nat. Cancer Inst. 103, 689-697 (2011).

15. Mitsuyama, Y. et al. Carcinoembryonic antigen and carbohydrate antigen 19-9 are prognostic predictors of colorectal cancer with unresectable liver metastasis. Oncol. Lett. 3, 767-771 (2012).

16. Tampellini, M. et al. The prognostic role of baseline CEA and CA 19-9 values and their time-dependent variations in advanced colorectal cancer patients submitted to first-line therapy. Tumour Biol. 36, 1519-1527 (2015).

17. Nozawa, H. et al. A high preoperative carbohydrate antigen $19-9$ level is a risk factor for recurrence in stage II colorectal cancer. Acta Oncol. 56, 634-638 (2017).

18. Scara, S.., Bottoni, P.., \& Scatena, R. C.. A19-9: biochemical and clinical aspects. Adv. Exp. Med Biol. 867, 247-260 (2015).

19. Dienstmann, R. et al. Prediction of overall survival in stage II and III colon cancer beyond TNM system: a retrospective, pooled biomarker study. Ann. Oncol. 28, 1023-1031 (2017).

20. Punt, C. J., Koopman, M. \& Vermeulen, L. From tumour heterogeneity to advances in precision treatment of colorectal cancer. Nat. Rev. Clin. Oncol. 14, 235-246 (2017).

21. Cohen, R. et al. BRAF-mutated colorectal cancer: what is the optimal strategy for treatment? Curr. Treat. Options Oncol. 18, 9 (2017).

22. Thomsen, $M$. et al. Interleukin- 6 and C-reactive protein as prognostic biomarkers in metastatic colorectal cancer. Oncotarget 7, 75013-75022 (2016).

23. Sorbye, $\mathrm{H}$. et al. Multicenter phase II study of Nordic fluorouracil and folinic acid bolus schedule combined with oxaliplatin as first-line treatment of metastatic colorectal cancer. J. Clin. Oncol. 22(1), 31-38 (2004).

24. Tveit, K. M. et al. Phase III trial of cetuximab with continuous or intermittent fluorouracil, leucovorin, and oxaliplatin (Nordic FLOX) versus FLOX alone in firstline treatment of metastatic colorectal cancer: the NORDIC-VII study. J. Clin. Oncol. 30, 1755-1762 (2012).

25. McMillan, D. C., Crozier, J. E., Canna, K., Angerson, W. J. \& McArdle, C. S. Evaluation of an inflammation-based prognostic score (GPS) in patients undergoing resection for colon and rectal cancer. Int J. Colorectal Dis. 22, 881-886 (2007).

26. Grenader, T. et al. Derived neutrophil lymphocyte ratio is predictive of survival from intermittent therapy in advanced colorectal cancer: a post hoc analysis of the MRC COIN study. Br. J. Cancer 114, 612-615 (2016).

27. Altman, D. G., McShane, L. M., Sauerbrei, W. \& Taube, S. E. Reporting Recommendations for Tumor Marker Prognostic Studies (REMARK): explanation and elaboration. PLoS Med. 9, e1001216 (2012).

28. Duffy, M. J. et al. Tumor markers in pancreatic cancer: a European Group on Tumor Markers (EGTM) status report. Ann. Oncol. 21, 441-447 (2010).

29. Prager, G. W. et al. Baseline carcinoembryonic antigen (CEA) serum levels predict bevacizumab-based treatment response in metastatic colorectal cancer. Cancer Sci. 105, 996-1001 (2014).

30. Yoshino, T. et al. Baseline carcinoembryonic antigen as a predictive factor of ramucirumab efficacy in RAISE, a second-line metastatic colorectal carcinoma phase III trial. Eur. J. Cancer 78, 61-69 (2017).

31. Rahbari, N. N. et al. Prognostic value of circulating endothelial cells in metastatic colorectal cancer. Oncotarget 8, 37491-37501 (2017).

32. Zhang, S. Y., Lin, M. \& Zhang, H. B. Diagnostic value of carcinoembryonic antigen and carcinoma antigen 19-9 for colorectal carcinoma. Int. J. Clin. Exp. Pathol. 8, 9404-9409 (2015)

33. Sanz-Garcia, E., Argiles, G., Elez, E. \& Tabernero, J. BRAF mutant colorectal cancer: prognosis, treatment, and new perspectives. Ann. Oncol. 28, 2648-2657 (2017).

34. Loupakis, F. et al. Initial therapy with FOLFOXIRI and bevacizumab for metastatic colorectal cancer. N. Engl. J. Med. 371, 1609-1618 (2014). 